\title{
Notched P-wave related to a conduction disturbance in a thoracic vein as a potential arrhythmogenic substrate of atrial fibrillation
}

\author{
Noriyuki Kobayashi ${ }^{1}$, Teppei Yamamoto $^{1}$, Yuhi Fujimoto ${ }^{1}$, Kenji Yodogawa ${ }^{1}$, Yu-ki \\ Iwasaki $^{1}$, and Wataru Shimizu ${ }^{1}$ \\ ${ }^{1}$ Nippon Medical School
}

May 21, 2020

\begin{abstract}
A 65-year-old male underwent a second ablation procedure for recurrent paroxysmal atrial fibrillation (AF). Twelve-lead ECG during sinus rhythm exhibited biphasic and split P-wave with a prolonged duration, which called notched P-wave (NPW). Radio-frequency applica-tion in the coronary sinus (CS) resulted AF termination and elimination of triggering ectopy with NPW disappearance. The CS musculature which played a principal role in triggering and maintaining AF formed the NPW. In patients with repetitive recurrent $\mathrm{AF}$ after the estab-lishment of the PV isolation who exhibit NPW during sinus rhythm, the CS might be one of the arrhythmogenic sources of AF.
\end{abstract}

\section{Keywords}

atrial fibrillation, notched P-wave, coronary sinus musculature

\section{Case description}

A 65-year-old man with a history of a circumferential PV isolation for paroxysmal atrial fibrillation (AF) and sick sinus syndrome underwent a second ablation procedure for recurrent paroxysmal AF. Twelve-lead ECG during sinus rhythm exhibited biphasic and split P-wave with a prolonged duration, which called notched Pwave (NPW, Figure 1A). Echocardiography revealed a normal LV systolic and diastolic function and an LA diameter of $31 \mathrm{~mm}$. Written informed consent was obtained before the procedure. An electrophysiological study was conducted in the fasting state and under deep conscious sedation. Although the first component of the NPW was formed by the total atrial activation including that of the LA appendage, the timing of the second component of the NPW was identical to that of the coronary sinus (CS) activation (Figure 1B). Activation mapping during distal CS pacing using an electroanatomical mapping system (CARTO3, Biosense Webster Inc., Diamond Bar, CA, USA) demonstrated 2 remote earliest activation sites in the LA near the anterolateral mitral annulus and inferior mitral annulus, respectively (Figure 2A). The shortest interval between the pacing stimulation spike to the LA was $45 \mathrm{~ms}$ suggesting selective local capture of the CS musculature (Figure 2B). Reconnections of the left superior PV and right inferior PV were also noted. AF was spontaneously initiated by an ectopic beat and repeated immediate recurrences occurred after electrical defibrillation even after the achievement of the PV isolation. Complex fractionated atrial electrograms were recorded at the LA lateral wall, LA appendage, and CS. Therefore, additional ablation at the mitral annulus and LA lateral wall was performed, however, it did not terminate the AF. The second component of the NPW remained unchanged after the ablation. Radio-frequency applications in distal portion of CS terminated AF and eliminated the ectopic beats (Figure 3) along with the disappearance of the second component of the Pwave (Figure 1B). Eventually, sinus rhythm was maintained, and AF was no longer induced by programmed stimulation with or without isoproterenol.

\section{Discussion}


Biphasic and split P-wave prolongation during sinus rhythm have been considered as an independent predictor of the development of $\mathrm{AF}$ and the recurrence of $\mathrm{AF}$ after $\mathrm{PV}$ isolation. ${ }^{1-3}$ The $\mathrm{P}$-wave prolongation is considered mainly to be a result of an electric conduction disturbance in the inter- or intra-atrium, and the second component of the P-wave is considered to be formed by delayed activation in the LA. ${ }^{4}$ Yanagisawa et al. ${ }^{1}$ reported that the disappearance of NPW after a previous PV isolation was predictor of a durable PV isolation and that the P-wave duration was shortened after the PV isolation in patients with NPW. Another report ${ }^{5}$ suggested that a prolonged conduction time from the right atrium to the CS predicted the recurrence of AF after catheter ablation. Therefore, a prolonged P-wave duration might suggest not only an inter- or intra-atrial conduction disturbance but also the presence of a conduction delay at the structures that have electrical connections to the LA, leading to the development of an atrial arrhythmogenic substrate. On the other hand, CS musculature possesses arrhythmogenicity for triggering AF as well as other thoracic vein and forms an arrhythmogenic substrate due to multiple myocardial connections to the LA for maintaining AF. ${ }^{6}$ Catheter ablation targeting a CS region is significantly associated with AF termination and a prolongation of AF cycle length. ${ }^{6}$ It has been reported that the additional ablation strategies beyond the PV isolation fail to reduce the $\mathrm{AF}$ recurrence rate. $^{7}$

From the anatomic and electrophysiologic viewpoint, the volume of cardiomyocytes in the CS musculature is small in comparison to that of the rest of atrium, and the electrical contribution of the CS musculature excitation on body surface ECG is limited. An electrical propagation from the proximal to distal CS during sinus rhythm is masked or fused by the LA excitation. However, in patients with a persistent left superior vena cava which has a substantial volume of cardiomyocytes, that causes changes in the $\mathrm{P}$ wave morphology, especially in the terminal portion of the positive/negative in lead III with significant left axis deviation. ${ }^{8}$ In addition, a significant conduction disturbance between the atrium and CS unmasks the excitation of the CS and also leads to a change in the $\mathrm{P}$ wave morphology. In our present case, the timing of the activation at LA appendage and lateral LA was identical to that of the first component of the P-wave, and the timing of the CS excitation was identical to that of the second component of the NPW even after the PV isolation (Figure 1B). The results of the termination and suppression of AF, and the disappearance of the second component of the P-wave with the RF application in the CS, strongly suggested that the second component of the P-wave was formed by the CS musculature activation and that the CS musculature played an important role in triggering $\mathrm{AF}$.

To the best of our knowledge, this is the first report showing an NPW that was formed by the activation in the CS musculature which played a principal role in triggering and maintaining AF. In patients with repetitive recurrent AF after the establishment of the PV isolation who exhibit NPW during sinus rhythm, the CS might be one of the arrhythmogenic sources of the AF.

\section{Acknowledgement}

The authors thank Mr. John Martin for his linguistic assistance.

\section{References}

1. Yanagisawa S, Inden $\mathrm{Y}$, Okamoto H, et al. Electrocardiogram characteristics of $\mathrm{P}$ wave associated with successful pulmonary vein isolation in patients with paroxysmal atrial fibrillation: Significance of changes in P-wave duration and notched P wave. Ann Noninvasive Electrocardiol. 2020;25:e12712.

2. Caldwell J, Koppikar S, Barake W, et al. Prolonged P-wave duration is associated with atrial fibrillation recurrence after successful pulmonary vein isolation for paroxysmal atrial fibrillation. J Interv Card Electrophysiol. 2014;39:131-138.

3. Hayashi H, Horie M. Biphasic P wave in inferior leads and the development of atrial fibrillation. J Arrhythm. 2015;31:376-380.

4. Antz M, Otomo K, Arruda M, et al. Electrical conduction between the right atrium and the left atrium via the musculature of the coronary sinus. Circulation. 1998;98:1790-1795. 
5. Kanemaru Y, Arima Y, Kaikita K, et al. Elongation of the high right atrium to coronary sinus conduction time predicts the recurrence of atrial fibrillation after radiofrequency catheter ablation. Int $\mathrm{J}$ Cardiol. 2020;300:147-153.

6. Haissaguerre M, Hocini M, Takahashi $\mathrm{Y}$, et al. Impact of catheter ablation of the coronary sinus on paroxysmal or persistent atrial fibrillation. J Cardiovasc Electrophysiol. 2007;18:378-386.

7. Verma A, Jiang CY, Betts TR, et al. Approaches to catheter ablation for persistent atrial fibrillation. N Engl J Med. 2015;372:1812-1822.

8. Ito-Hagiwara K, Iwasaki YK, Hayashi M, et al. Electrocardiographic characteristics in the patients with a persistent left superior vena cava. Heart Vessels. 2019;34:650-657.

Figure legends

Figure 1 A: Surface 12-lead ECG showing sinus rhythm with notched P-wave (left, pre-procedure) and without notched P-wave (right, post-procedure). B: The delayed coronary sinus (CS) potentials are identical only to the second component of the notched $\mathrm{P}$ wave (arrow). C: The notched $\mathrm{P}$-wave disappeared after radio frequency applications in the CS. The amplitudes of the CS potentials were lower in intra-CS electrograms of post ablation. $\mathrm{LA}=$ left atrium.

Figure 2 A: Left lateral view of the activation map. Theblue circular tags indicate the earliest activation sites and theblue star tag indicates the site where pacing was performed.B: Pacing from the distal pair of electrodes of the catheter placed in the coronary sinus (CS) exhibiting a potential by $45 \mathrm{~ms}$ delay from the stimulation spike on the mapping catheter (Map) placed on the anterolateral wall of the mitral annulus. $\mathrm{ABL}=$ ablation catheter.

Figure 3 A: Recordings during the radiofrequency $(\mathrm{RF})$ energy application. The arrhythmia was terminated by an RF application at a distal site in coronary sinus (CS). B: Fluoroscopic image during the successful RF application. The CS catheter was pulled back in order to allow the ablation catheter (ABL) to be cannulated into the distal CS. Eso = esophagus; LAA = left atrial appendage.

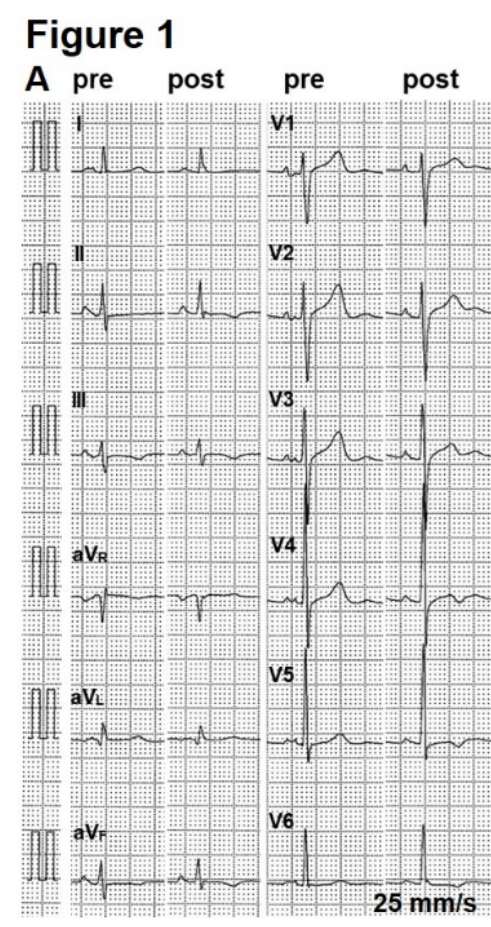

B
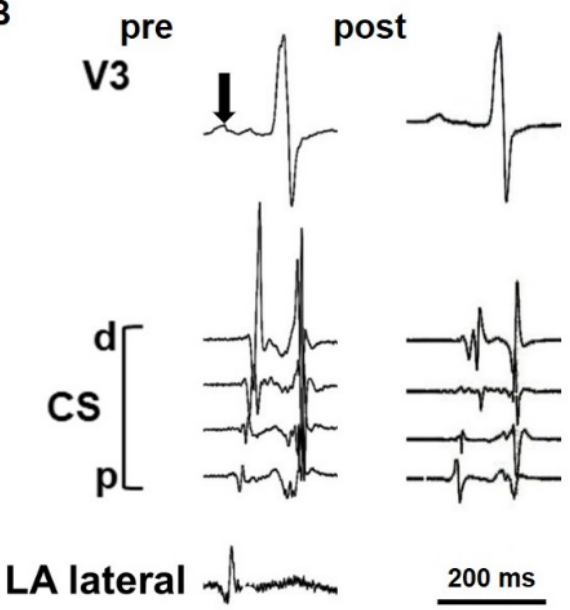
Figure 2
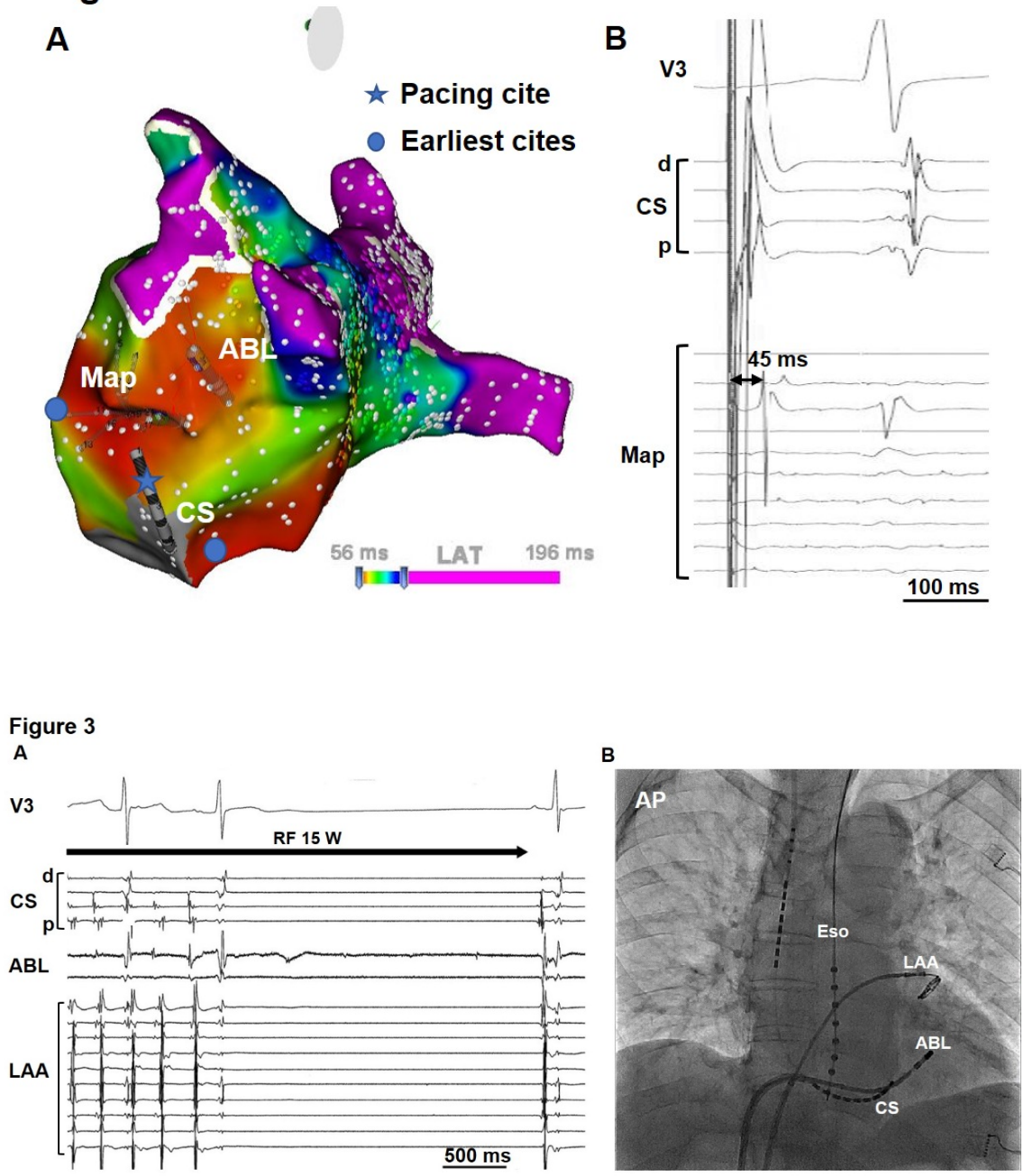